\title{
String Stability Analysis of Homogeneous Linear Unidirectionally Connected Systems with nonzero Initial Conditions
}

\author{
Steffi Klinge ${ }^{\dagger}$ and Richard H. Middleton* \\ Hamilton Institute, NUI Maynooth, Maynooth, Co. Kildare, Ireland \\ E-mail: ${ }^{\dagger}$ mail@steffi-klinge.de $\quad{ }^{*}$ richard.middleton@nuim.ie
}

\begin{abstract}
This paper investigates string stability issues in homogeneous strings of strictly proper feedback control systems. We consider initial condition problems with unidirectional nearest neighbour communications, using only linear systems with two integrators in the loop. We define string stability as a type of stability that is uniform with respect to the string length. We show conditions under which the $L_{2}$-norm and the $L_{\infty}$-norm of states are bounded and derive a formula for the minimal time headways required to guarantee string stability.
\end{abstract}

Keywords - Control Systems, Communication Systems and Networks

\section{INTRODUCTION}

Since commercial and private vehicle traffic increased significantly during the last decades a growing number of researchers are focusing on 'platooning'. This special field of formation control concentrates on finding suitable methods to control a linear string of automobiles driving in a column. Among the desirable features of platooning are for example higher traffic throughput, the safety gain and reduced fuel consumption.

A lot of work has been done for the simplest form of platoon control where a constant distance between the vehicles is required, e.g. [1-5]. To simplify communication requirements, and to achieve a controller design which does not depend on the string length, we consider the case where the automobiles are equipped with a local controller based on sensing the distance to the preceding vehicle. If the dynamics of the vehicle and controller are also independent of the location in the string, the string is called 'homogeneous'.

Since every controller only uses the information of the separation to its predecessor the system structure will be triangular and studying the sta-

The authors would like to thank the Science Foundation of Ireland for supporting this work with grant $07 / \mathrm{RPR} / \mathrm{I} 177$. bility of the system is relatively easy. Thus, for a fixed string length, and appropriately designed local controllers, asymptotic and input-output stability can be guaranteed. However, in some cases, these forms of stability are not uniform with respect to string length, and as the string length grows, the disturbance response may grow without bound. That effect is often called 'string instability'.

Different definitions of string stability have been used, including mostly input-output formulations and some rare initial conditions and state space formulations, [6]. Due to simpler analysis most research is based around the Euclidean norm, [7,8] but definitions involving the maximum norm can also be found, [9].

It is not possible to achieve string stability in a homogeneous string of strictly proper feedback control systems with nearest neighbour communications if only linear systems with two integrators in the open loop and a constant inter-vehicle spacing is required, [3]. It has also been shown that this result is independent of the particular linear controller design, $[7,10]$.

Techniques that yield string stability include: Introduction of a speed dependent inter-vehicle spacing policy (also called 'time headway pol- 


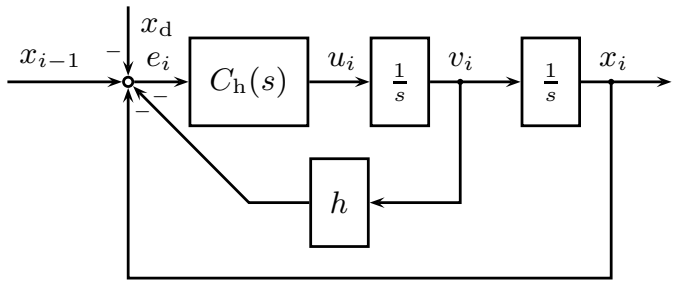

Figure 1: Block diagram of the linear system with time headway

icy'), [11], implementing heterogeneous strings, i.e. the particular controller depends on the position within the string, $[8,12]$, and using nonlinear spacing policies, [13].

This paper presents some of the results in [5]. We will focus on a homogeneous string of linear control systems with two integrators in the open loop using unidirectional nearest neighbour communication. In Section II we will clarify the notation and describe the behaviour of the system as a state space model. After showing string instability for the case where zero or a small time headway is used in Section III, we will derive a formula for the minimal time headway required and prove string stability when using a sufficiently large time headway in Section IV. The results will be illustrated by an example and simulations in Section V.

\section{PRELIMINARIES}

We wish to discuss the stability of a simple chain of $N$ vehicles where all but the first should keep a fixed distance $x_{\mathrm{d}}$ to their predecessor. The first car follows a given trajectory. We will choose the same vehicle model and the same linear controller to stabilise the plant (i.e. to force the position error to zero, $e_{i} \rightarrow 0$ ) for every subsystem, i.e. every car. Since the trajectory the first vehicle must follow is a ramp with slope $v_{\mathrm{r}}$, we must have at least two integrators in the open loop of every subsystem to force the steady state error signals to zero.

We will choose a vehicle model and an appropriate controller design such that the open loop transfer function of every subsystem has exactly two poles at the origin, e.g. two integrators in the plant. It has been shown that such a homogeneous string of linear, time invariant subsystems with two integrators in the open loop is string unstable if a fixed distance $x_{\mathrm{d}}$ between the vehicles is required, $[3,7]$. That is a small disturbance at the beginning of the string amplifies without bound while propagating down string. However, using a time headway $h$ (i.e. an additional velocity $v_{i}$ dependent term, see Figure 1) can guarantee string stability and ensure bounded error signals $e_{i}=x_{i-1}-x_{i}-x_{\mathrm{d}}-h v_{i}$ independent of the string length $N,[11]$.
We want to transform the state space with the physical states of the system such as position $x_{i}$, velocity $v_{i}$, controller input $u_{i}$, and the controller states $x_{\mathrm{c}_{j_{i}}}$ into deviation coordinates $\xi_{i} \stackrel{t \rightarrow \infty}{\longrightarrow} 0$ such that they can be described by

$\dot{\xi}_{i}=A \xi_{i}+b c^{\mathrm{T}} \xi_{i-1}, \quad \forall i>2$

$\dot{\xi}_{1}=A \xi_{1}$

Therefore, we choose $\xi_{i}=X_{i-1}-X_{i}$ with $X_{i}^{\mathrm{T}}=\left(\begin{array}{lllll}e_{i} & v_{i} & x_{\mathrm{c}_{1_{i}}} & x_{\mathrm{c}_{2_{i}}} & \cdots\end{array}\right)$. Details of the necessary transformation can be found in [5].

In vector form, we can write

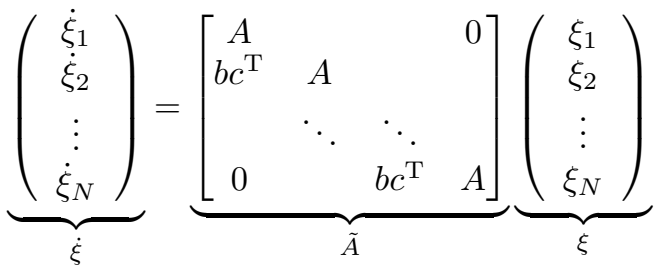

Since measurement noise and disturbances are neglected for simplicity, $\xi(t)$ only depends on the initial conditions $\xi(0)$ :

$\xi(t)=\mathcal{L}^{-1}\left\{(s I-\tilde{A})^{-1}\right\} \xi(0)$

Note that the local, single closed loop transfer function $c^{\mathrm{T}}(s I-A)^{-1} b=\Gamma(s)$, can be written as $\Gamma(s)=\frac{1}{Q(s)} T(s)$, with $Q(s)=h s+1$ and $T(s)$ being the complementary sensitivity function of the system. Assume $C_{\mathrm{h}}(s)$ is designed as a function of $h$ to keep $T(s)=\frac{C(s)}{s^{2}+C(s)}$ independent of $h$.

We consider the following definitions for string stability for nonzero initial conditions:

Definition 1 ( $L_{2}$-String Stability) Consider a string of $N$ dynamic systems of dimension $n$ described by

$\dot{\xi}_{i}=f\left(\xi_{i}, \xi_{i-1}\right) \quad \forall 1<i \leq N, \quad \dot{\xi}_{1}=f\left(\xi_{1}, 0\right)$

where $N \in \mathbb{N}, \xi_{i} \in \mathbb{R}^{n}, f: \mathbb{R}^{n} \times \mathbb{R}^{n} \rightarrow \mathbb{R}^{n}$, $f(0,0)=0$. The origin $\xi_{i}=0, \forall i$ of $(5)$ is $L_{2}$ string stable if given any $k \in\{1,2, \ldots, N\}$ and any $\epsilon>0$ there exists a $\delta>0$, such that

$$
\begin{aligned}
\xi_{i}(0)= & 0, i \neq k \text { and }\left\|\xi_{k}(0)\right\|_{2}<\delta \\
& \Rightarrow \sup _{i}\left\|\xi_{i}(t)\right\|_{\mathrm{L}_{2}}<\epsilon
\end{aligned}
$$

where $\delta$ is independent of the string length $N$.

Definition 2 ( $L_{\infty}$-String Stability) Consider a string of dynamic systems as described in (5). The origin $\xi_{i}=0, \forall i$ of $(5)$ is $L_{\infty}$-string stable if given any $k \in\{1,2, \ldots, N\}$ and any $\epsilon>0$ there exists a $\delta>0$, such that

$$
\begin{aligned}
\xi_{i}(0)= & 0, i \neq k \text { and }\left\|\xi_{k}(0)\right\|_{\infty}<\delta \\
& \Rightarrow \sup _{i}\left\|\xi_{i}(t)\right\|_{\mathrm{L}_{\infty}}<\epsilon
\end{aligned}
$$

where $\delta$ is independent of the string length $N$. 
Thus, we focus on a special class of initial conditions, where only the initial values of one single subsystem $k$ are non zero. If the Euclidean norm, or the maximum norm of $\xi_{k}(0)$ is bounded, we require the $L_{2}$-norm, or the $L_{\infty}$-norm of the states of the $i$ th subsystem to be bounded for every subsystem $i$ within the string, respectively.

\section{STRING INSTABILITY FOR SMALL TIME HEADWAYS}

The behaviour of $\xi(t)$ is described by (4) where $(s I-\tilde{A})^{-1}$ can be written elementwise as

$$
(s I-\tilde{A})_{i j}^{-1}= \begin{cases}0, & \text { for } i<j \\ (s I-A)^{-1}, & \text { for } i=j \\ (s I-A)^{-1} b \Gamma^{(i-j-1)} & \text { for } i>j \\ \cdot c^{\mathrm{T}}(s I-A)^{-1}, & \text {. }\end{cases}
$$

Equations (4) and (6) lead us to

$$
\xi_{i}(t)=\left\{\begin{array}{lr}
0, & \text { for } i<k \\
\mathcal{L}^{-1}\left\{(s I-A)^{-1}\right\} \xi_{k}(0), & \text { for } i=k \\
\mathcal{L}^{-1}\left\{(s I-A)^{-1} b \Gamma^{(i-k-1)}\right. & \\
\left.\cdot c^{\mathrm{T}}(s I-A)^{-1}\right\} \xi_{k}(0), & \text { for } i>k
\end{array}\right.
$$

\section{a) $\quad L_{2}$-String Instability}

To satisfy Definition 1 the $L_{2}$-norm of every set of states of every subsystem must be bounded for any bounded $\left\|\xi_{k}(0)\right\|_{2}$. Therefore the $L_{2}$-norm of every combination of states of $\xi_{i}(t)$ must also be bounded.

For simplicity we will now choose $\xi_{i}^{\prime}(t)=c^{\mathrm{T}} \xi_{i}(t)$ and $\xi_{k}(0)=b$ and show that the $L_{2}$-norm of $\xi_{i}^{\prime}(t)$ grows without bound if $i$ increases and $i>k$.

For $i>k$ the $L_{2}$ norm of $\xi_{i}^{\prime}(t)$ is given by

$$
\left\|\xi_{i}^{\prime}(t)\right\|_{\mathrm{L}_{2}}=\left\|\mathcal{L}^{-1}\left\{\Gamma^{(i-k+1)}(s)\right\}\right\|_{\mathrm{L}_{2}}
$$

Using Parseval's relation, we can calculate the square of the $L_{2}$-norm of $\xi_{i}^{\prime}(t)$ for the initial condition given

$$
\begin{aligned}
\left\|\xi_{i}^{\prime}(t)\right\|_{\mathrm{L}_{2}}^{2} & =\frac{1}{2 \pi} \int_{-\infty}^{\infty}\left|\xi_{i}^{\prime}(j \omega)\right|^{2} \mathrm{~d} \omega \\
& =\frac{1}{2 \pi} \int_{-\infty}^{\infty}\left|\Gamma^{(i-k+1)}(j \omega)\right|^{2} \mathrm{~d} \omega
\end{aligned}
$$

It is known that the absolute value of the complementary sensitivity function of a single subsystem, $\Gamma(j \omega)$, is greater than $1+\epsilon$ for a range of frequencies $\omega \in\left(\omega_{-}, \omega_{+}\right)$, if zero or a small time headway is used, $[3,5,7]$.

$$
\int_{R}^{\text {Since }} f(x) \mathrm{d} x \geq \int_{T \subset R} f(x) \mathrm{d} x
$$

for any non-negative function $f(x)$, we can bound (9) by

$$
\begin{aligned}
\left\|\xi_{i}^{\prime}(t)\right\|_{\mathrm{L}_{2}}^{2} & \geq \frac{1}{2 \pi} \int_{\omega_{-}}^{\omega_{+}}\left|\Gamma^{(i-k+1)}(j \omega)\right|^{2} \mathrm{~d} \omega \\
& \geq \frac{1}{2 \pi}\left(\omega_{+}-\omega_{-}\right)(1+\epsilon)^{2(i-k+1)}
\end{aligned}
$$

So $\left\|\xi_{i}^{\prime}(t)\right\|_{\mathrm{L}_{2}}$ will grow as $i$ increases and the system cannot be $L_{2}$-string stable in the sense of Definition 1.

\section{b) $L_{\infty}$-String Instability}

We will now expand the argument above and show, that the system cannot be $L_{\infty}$-string stable either. Note that since $\Gamma(s)$ is analytic in the closed right half plane, it is continuous there. Therefore, there exist an $\eta>0$ and an $\epsilon_{\eta} \in(0, \epsilon)$, such that $|\Gamma(\eta+j \omega)| \geq 1+\epsilon_{\eta}$ for all $\omega \in\left[\omega_{-}, \omega_{+}\right]$. Therefore the following analogy to (9) and (11) holds

$$
\begin{aligned}
& \frac{1}{2 \pi} \int_{-\infty}^{\infty}\left|\xi_{i}^{\prime}(\eta+j \omega)\right|^{2} \mathrm{~d} \omega \\
& \quad=\frac{1}{2 \pi} \int_{-\infty}^{\infty}|\Gamma(\eta+j \omega)|^{2(i-k+1)} \mathrm{d} \omega \\
& \quad \geq \frac{1}{2 \pi}\left(\omega_{+}-\omega_{-}\right)\left(1+\epsilon_{\eta}\right)^{2(i-k+1)}
\end{aligned}
$$

Using Parseval's Theorem and frequency shifting, we obtain

$$
\begin{aligned}
\frac{1}{2 \pi} \int_{-\infty}^{\infty}\left|\xi_{i}^{\prime}(\eta+j \omega)\right|^{2} \mathrm{~d} \omega & =\int_{0}^{\infty} \xi_{i}^{\prime 2}(t) \mathrm{e}^{-2 \eta t} \mathrm{~d} t \\
& \leq \frac{1}{2 \eta}\left\|\xi_{i}^{\prime}(t)\right\|_{\mathrm{L}_{\infty}}^{2}
\end{aligned}
$$

Combining (13) and (14), we see that

$\left\|\xi_{i}^{\prime}(t)\right\|_{\mathrm{L}_{\infty}} \geq\left(1+\epsilon_{\eta}\right)^{(i-k+1)} \sqrt{\frac{\eta}{\pi}\left(\omega_{+}-\omega_{-}\right)}$

and therefore the system cannot be $L_{\infty}$-string stable according to Definition 2.

However, if a sufficiently large time headway $h$ is chosen $L_{2}$-string stability and $L_{\infty}$-string stability can be guaranteed as we will show below.

\section{StRING STABILITY FOR SUFFICIENT TIME HEADWAYS}

\section{a) $L_{2}$-String Stability}

Since we have seen above that $\|\Gamma(j \omega)\|>1$ will provoke string instability, we will now choose a sufficiently large time headway $h$ such that $|\Gamma(j \omega)| \leq 1$ 
for all $\omega$.

$$
\begin{aligned}
|\Gamma(j \omega)|^{2} & =\frac{1}{1+\omega^{2} h_{2}^{2}}|T(j \omega)|^{2} \leq 1 \quad \forall \omega \\
h_{2} & :=\sqrt{\max _{\omega}\left(\frac{|T(j \omega)|^{2}-1}{\omega^{2}}\right)}
\end{aligned}
$$

where $T(s)$ is the complementary sensitivity function for the system with zero time headway. Thus, picking a time headway greater or equal the minimal time headway $h_{2}$, will ensure $|\Gamma(j \omega)| \leq 1$ for all $\omega$.

Again, $\xi_{i}(t)$ is described by (7) and we analyse the $L_{2}$-norm of $\xi_{i}(t)$ for $i>k$ and use of Parseval's Theorem

$$
\begin{aligned}
\left\|\xi_{i}(t)\right\|_{\mathrm{L}_{2}}= & \| \mathcal{L}^{-1}\left\{(s I-A)^{-1} b\right. \\
& \left.\cdot \Gamma^{(i-k-1)} c^{\mathrm{T}}(s I-A)^{-1}\right\} \xi_{k}(0) \|_{\mathrm{L}_{2}} \\
\leq & \left\|(s I-A)^{-1} b \Gamma^{(i-k-1)} c^{\mathrm{T}}(s I-A)^{-1}\right\|_{\mathrm{H}_{2}} \\
& \cdot\left\|\xi_{k}(0)\right\|_{2} \\
\leq & \left\|(s I-A)^{-1} b\right\|_{\mathrm{H}_{\infty}}\|\Gamma\|_{\mathrm{H}_{\infty}}^{(i-k-1)} \\
& \cdot\left\|c^{\mathrm{T}}(s I-A)^{-1}\right\|_{\mathrm{H}_{2}}\left\|\xi_{k}(0)\right\|_{2}
\end{aligned}
$$

Since $A$ is Hurwitz the values of $\left\|(s I-A)^{-1} b\right\|_{\mathrm{H}_{\infty}}$ and $\left\|c^{\mathrm{T}}(s I-A)^{-1}\right\|_{\mathrm{H}_{2}}$ are some finite numbers. With $h \geq h_{2},\|\Gamma(j \omega)\|=\sup _{\omega}|\Gamma(j \omega)|=1$ and for bounded initial conditions $\left\|\xi_{k}(0)\right\|_{2}<\delta,\left\|\xi_{i}(t)\right\|_{\mathrm{L}_{2}}$ is bounded independently of the string length $N$ or the position within the string $i$.

\section{b) $\quad L_{\infty}$-String Stability}

Since the dynamics of the system is described by (7), the $L_{\infty}$-norm of $\xi(t)$ is the maximum of all $\left\|\xi_{i}(t)\right\|_{\mathrm{L}_{\infty}}$. Thus, in order to satisfy $L_{\infty}$-string stability according to Definition 2 all states in $\xi(t)$ need to be bounded. The product of inverse Laplace-transforms is the same as the convolution of the corresponding impulse responses:

$\xi_{i}(t)=\left\{\begin{array}{lr}0, & \text { for } i<k \\ \alpha(t) \xi_{k}(0), & \text { for } i=k \\ \phi(t) * \gamma(t)^{*(i-k-1)} * c \alpha(t) \xi_{k}(0), & \text { for } i>k\end{array}\right.$

where $\alpha(t)=\mathcal{L}^{-1}\left\{(s I-A)^{-1}\right\}$, $\phi(t)=\mathcal{L}^{-1}\left\{(s I-A)^{-1} b\right\}, \quad \gamma(t)=\mathcal{L}^{-1}\{\Gamma(s)\}$, and $\gamma(t)^{* i}=\underbrace{\gamma(t) * \gamma(t) * \ldots * \gamma(t)}_{i \text { times }}$.

Since $A$ is Hurwitz, the $L_{\infty}$-norm of $\alpha(t) \xi_{k}(0)$ is bounded for bounded initial conditions $\xi_{k}(0)$, the norm of $\phi(t) * \gamma(t)^{*(i-k-1)} * c \alpha(t) \xi_{k}(0)$ for $i>k$ must be bounded independently of $i$ or $N$ to satisfy
Definition 2.

$$
\begin{aligned}
\left\|\xi_{i}(t)\right\|_{\mathrm{L}_{\infty}} & =\left\|\phi(t) * \gamma(t)^{*(i-k-1)} * c \alpha(t) \xi_{k}(0)\right\|_{\mathrm{L}_{\infty}} \\
& \leq\left\|\phi(t) * \gamma(t)^{*(i-k-1)} * c \alpha(t)\right\|_{\mathrm{i}_{\infty}}\left\|\xi_{k}(0)\right\|_{\infty}
\end{aligned}
$$

Because the controller is designed in a way that $A$ is Hurwitz the impulse responses $\alpha(t)$ and $\phi(t)$ are bounded. Thus the induced $L_{\infty}$-norm will depend on the norm of $\gamma^{*(i-k-1)}(t)$ which is always less or equal to the norm of $\gamma(t)$ to the power of $(i-k-1)$ $\left(\left\|\gamma^{*(i-k-1)}(t)\right\| \leq\|\gamma(t)\|^{(i-k-1)}\right)$.

Note that the induced $L_{\infty}$-norm of an operator is the $L_{1}$-norm of its impulse response. Hence, one way to insure that the system is $L_{\infty}$-string stable is to require $\|\gamma(t)\|_{L_{1}}$ to be less or equal than 1. That is the same as requiring a monotonically non decreasing step response or equivalently a non negative impulse response.

Since the complementary sensitivity function has changed from $T(s)$ to $\Gamma(s)=Q(s)^{-1} T(s)$ by introducing the time headway the corresponding impulse response changed as well from $\gamma_{0}(t)=\mathcal{L}^{-1}\{T(s)\}$ to $\gamma(t)=\mathcal{L}^{-1}\{\Gamma(s)\}$.

$$
\begin{aligned}
\gamma(t) & =\mathcal{L}^{-1}\left\{\frac{1}{h s+1} T(s)\right\} \\
& =\frac{1}{h} \mathrm{e}^{-\frac{t}{h}} \int_{0}^{t} \mathrm{e}^{\frac{\tau}{h}} \gamma_{0}(\tau) \mathrm{d} \tau
\end{aligned}
$$

Because $\frac{1}{h} e^{-\frac{t}{h}}>0$ the integral over $e^{\frac{t}{h}} \gamma_{0}(t)$ needs to be greater or equal then zero.

$\gamma(t)=\frac{1}{h} \mathrm{e}^{-\frac{t}{h}} \int_{0}^{t} \mathrm{e}^{\frac{\tau}{h}} \gamma_{0}(\tau) \mathrm{d} \tau \geq 0 \Leftrightarrow \underbrace{\int_{0}^{t} \mathrm{e}^{\frac{\tau}{h}} \gamma_{0}(\tau) \mathrm{d} \tau}_{\bar{\gamma}(t)} \geq 0$

If possible we need to find a $h_{\infty}$ which is the minimal time headway $h$ satisfying (22). Thus, every $h \geq h_{\infty}$ guarantees $L_{\infty}$-string stability.

Since $\gamma(t)$ is continuous we only need to make sure that all local maxima and minima of $\bar{\gamma}(t)$ are greater or equal then zero. Local extrema appear if the derivative of $\bar{\gamma}(t)$ is zero

$\frac{\mathrm{d}}{\mathrm{d} t}(\bar{\gamma}(t))=e^{\frac{t}{h}} \gamma_{0}(t)=0 \quad \Longleftrightarrow \quad \gamma_{0}(t)=0$

Thus, we must assure that $\gamma_{0}(t)$ changes sign only a finite number of times by requiring that the dominant pole of $T(s)$ is real. Then $\bar{\gamma}(t)$ must be non negative at the zero crossings of $\gamma_{0}(t)$. If $\bar{\gamma}(t)$ is non negative at all zero crossings of $\gamma_{0}(t)$ the impulse response $\gamma(t)$ will be non negative for all $t>0$. 


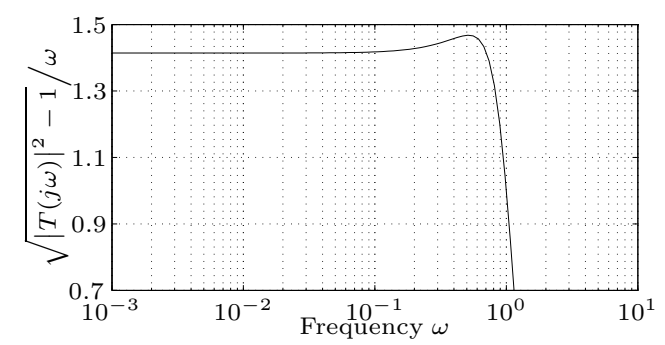

Figure $2: \sqrt{|T(j \omega)|^{2}-1} / \omega$ for $T(s)=\frac{s+1}{s^{2}+s+1}$

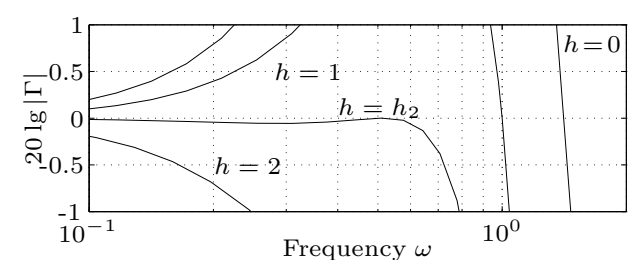

Figure 3: $\lg |\Gamma(j \omega)|$ for different time headways

Therefore we can guarantee $L_{\infty}$-string stability according to Definition 2 by using a time headway $h \geq h_{\infty}$.

Note that under some mild conditions $h \geq h_{2}$ is also sufficient for $L_{\infty}$-string stability, [5].

Swaroop et al. established a different result focusing on the poles and zeros of $T(s)$ to assure a positive impulse response in [14].

\section{EXAMPLE AND SIMULATION RESULTS}

In this section we will illustrate our results by presenting some simulations of a simple example system. We will choose $T(s)=\frac{s+1}{s^{2}+s+1}$ as the complementary sensitivity function of the system.

First, we need to find the minimal time headway that gives $L_{2}$-string stability according to (17). For $T(s)=\frac{s+1}{s^{2}+s+1}$ the curve of $\sqrt{|T(j \omega)|^{2}-1} / \omega$ is shown in Figure 2. The maximum is achieved at $\omega \approx 0.5$ and $h_{2} \approx 1.47$. In Figure 3 we can observe how the magnitude of $\Gamma(j \omega)$ changes with the time headway $h$. If no or a small time headway (e.g. $h=1)$ is used $|\Gamma(j \omega)|$ is larger than one for a range of frequencies. However, as soon as $h \geq h_{2}$, $|\Gamma(j \omega)| \leq 1$ for all $\omega$.

Impulse responses for different values of $h$ are shown in Figure 4 . Only $h \geq h_{\infty}=2.42$ guarantees a non negative impulse response.

A string of 20 vehicles with the complementary sensitivity function $\Gamma(s)=\frac{1}{h s+1} T(s)$ and non zero initial conditions in the first subsystem has been simulated for different time headways $h$.

The behaviour of the error signals $e_{i}(t)$ without using a time headway is shown in Figure 5. Appar-

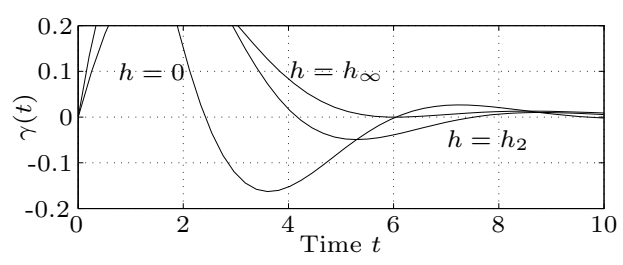

Figure 4: $\gamma(t)$ for different time headways

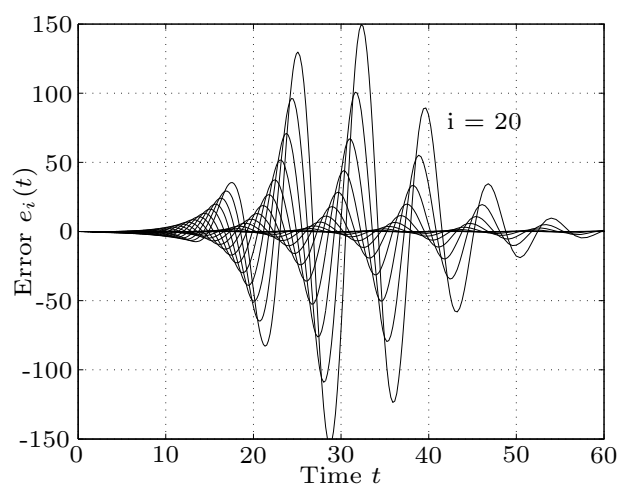

Figure 5: Error signals $e_{i}(t)$ for $h=0$

ently the error signal $e_{i}(t)$ increases as $i$ increases and the system therefore is string unstable.

Using a small time headway of $h<h_{2}$ improves the performance of the string significantly, shown in Figure 6. However, the string is not string stable since the norm of the error signal $e_{i}(t)$ grows as $i$ increases.

Simulations with $h=h_{2}$ can be observed in Figure 7 . The norm of the error signals $e_{i}(t)$ is now bounded independently of the string length $N$ and the position within in the string $i$. However, it is hard to detect string stability in a simulation of 20 vehicles only.

The effect of a sufficiently large time headway can also be observed in Figure 8. Although $2<2.42=h_{\infty}$ the $L_{\infty}$-norm of $e_{i}$ does not grow with $i$ and the system is $L_{\infty}$-string stable.

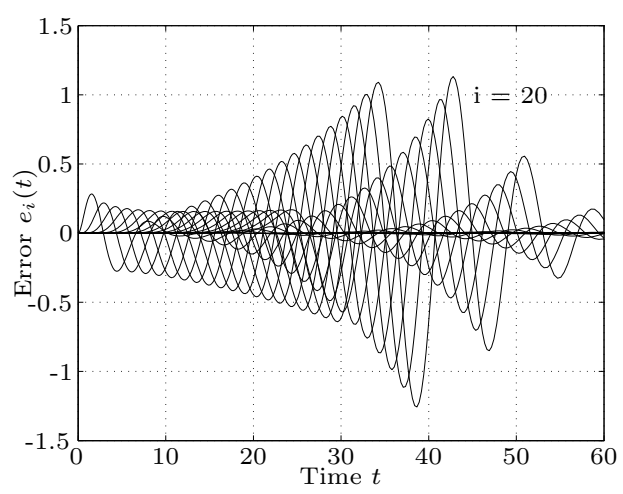

Figure 6: Error signals $e_{i}(t)$ for $h=1$ 


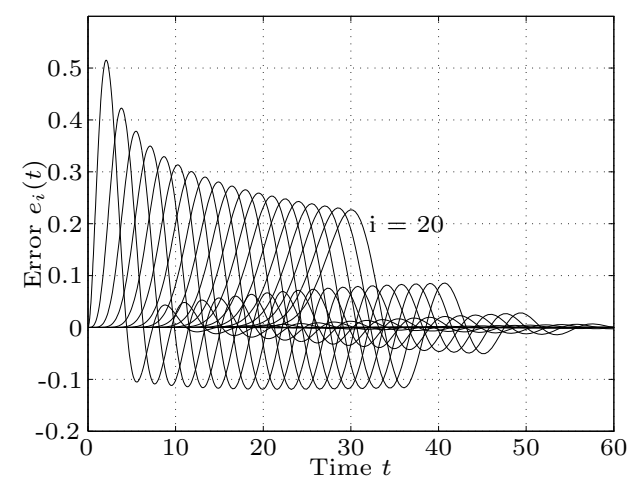

Figure 7: Error signals $e_{i}(t)$ for $h=h_{2}$

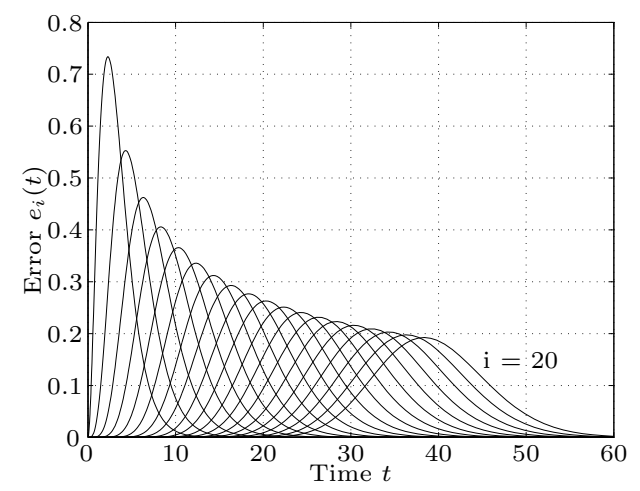

Figure 8: Error signals $e_{i}(t)$ for $h=2$

\section{CONCLUSions AND FUture DiRECTIONS}

In this paper we have discussed $L_{2}$-string stability and $L_{\infty}$-string stability of a class of homogeneous strings of linear, time invariant control systems with two integrators in the open loop using unidirectional nearest neighbour communication. We showed that zero or a small time headway provokes string instability. A formula for the minimal time headway $h_{2}$, or $h_{\infty}$ to guarantee $L_{2}$-string stability, or $L_{\infty}$-string stability was derived and string stability was proved for $h \geq h_{2}, h \geq h_{\infty}$.

As for future directions, it would be interesting to extend the results presented to more general cases. That could be analyzing heterogeneous systems, or bidirectional controller designs.

\section{REFERENCES}

[1] W. Levine and M. Athans, "On the optimal error regulation of a string of moving vehicles," IEEE Transactions on Automatic Control, vol. AC-11, no. 3, pp. 355-361, 1966.

[2] K. C. Chu, "Decentralized control of highspeed vehicular strings," Transportation Science, vol. 8, no. 4, pp. 361 - 384, 1974.

[3] S. Sheikholeslam and C. Desoer, "Longitudinal control of a platoon of vehicles," in Proceedings of the American Control Conference, 1990, pp. 291-297.
[4] D. Swaroop, "String stability of interconnected systems: An application to platooning in automated highway systems," Ph.D. dissertation, University of California, Berkeley, CA, 1994.

[5] S. Klinge, "Stability issues in distributed systems of vehicle platoons," Master's thesis, Otto-von-Guericke-University Magdeburg, http://www.hamilton.ie/publications.htm, 2008.

[6] D. Swaroop and J. Hedrick, "String stability of interconnected systems," IEEE Transactions on Automatic Control, vol. 41, no. 3, pp. 349 - 357, 1996.

[7] P. Seiler, A. Pant, and K. Hedrick, "Disturbance propagation in vehicle strings," IEEE Transactions on Automatic Control, vol. 49, no. 10, pp. 1835 - 1841, 2004.

[8] I. Lestas and G. Vinnicombe, "Scalability in heterogeneous vehicle platoons," in Proceedings of the American Control Conference, 2007, pp. 4678-4683.

[9] J. Eyre, D. Yanakiev, and I. Kanellakopoulos, "A simplified framework for string stability analysis of automated vehicles," Vehicle Systems Dynamic, vol. 30, no. 5, pp. 375-405, 1998.

[10] P. Barooah and J. Hespanha, "Error amplification and disturbance propagation in vehicle strings with decentralized linear control," in Proceedings of the 44th IEEE Conference on Decision and Control, and the European Control Conference 2005, 2005, pp. 4964-4969.

[11] C. Chien and P. Ioannou, "Automatic vehicle following," in Proceedings of the American Control Conference, 1992, pp. 1748-1752.

[12] M. Khatir and E. Davison, "Bounded stability and eventual string stability of a large platoon of vehicles using non-identical controllers," in Proceedings of the 43rd Conference on Decision \& Control, 2004, pp. 1111-1116.

[13] D. Yanakiev and I. Kanellakopoulos, "Nonlinear spacing policies for automated heavyduty vehicles," IEEE Transactions on Vehicular Technology, vol. 47, no. 4, pp. 1365-1377, 1998.

[14] D. Swaroop and D. Niemann, "Some new results on the oscillatory behavior of impulse and step responses for linear time-invariant systems," in Proceedings of the 35th Conference on Decision \& Control, 1996. 